\title{
REPRESENTATION OF CERTAIN CLASSES OF DISTRIBUTIVE LATTICES BY SECTIONS OF SHEAVES
}

\section{U. MADDANA SWAMY and P. MANIKYAMBA}

\author{
Mathematics Department \\ Andhra University \\ Waltair 530003 \\ INDIA
}

(Received March 13, 1979 and in revised form July 9, 1979)

ABSTRACT. Epstein and Horn ([6]) proved that a Post algebra is always a $\mathrm{P}$-algebra and in a P-algebra, prime ideals lie in disjoint maximal chains. In this paper it is shown that a P.algebra $L$ is a Post algebra of order $n \geq 2$, if the prime ideals of $\mathrm{L}$ lie in disjoint maximal chains each with $\mathrm{n}-1$ elements. The main tool used in this paper is that every bounded distributive lattice is isomorphic with the lattice of all global sections of a sheaf of bounded distributive lattices over a Boolean space. Also some properties of P-algebras are characterized in terms of the stalks.

KEY WORDS AND PHRASES. POSt Algebra, P-algebra, B-algebra, Heyting Algebra, Stone Lattice, Boolean Space, Sheas of Distributive Lattices Over a Boolean Space, Prime Ideals Lie in Disjoint Maximal Chains, Regular Chain Base. 1980 Mathematics Subject Classification Codes: Primary 06A35, Secondary 18 F 20 . 
1. INTRODUCTION.

Epstein ([5]) proved that in a Post algebra of order $n \geq 2$ prime ideals lie in disjoint maximal chains each with $n-1$ elements. He has also proved that if $\mathrm{L}$ is a finite distributive lattice and prime ideals of $\mathrm{L}$ lie in disjoint maximal chains each with $n-1$ elements, then $L$ is a Post algebra of order n. Epstein and Horn ([6]) have shown that a Post algebra is always a Palgebra and in a P-algebra prime ideals lie in disjoint maximal chains. It is the main theme of this paper that a P-algebra L is a Post algebra of order $\mathrm{n} \geq 2$, if the prime ideals of $\mathrm{L}$ lie in disjoint maximal chains each with $\mathrm{n}-1$ elements.

The main tool used in this paper is the fact that every bounded distributive lattice is isomorphic with the lattice of all global sections of a sheaf of bounded distributive lattices over a Boolean space ([15] and [9]). It is well known that a P-algebre is always a (double) Heyting algebra, a (double) L-algebra, a pseudocomplemented lattice, a Stone lattice and a normal lattice. We characterize these properties of P-algebras in detail in terms of the stalks of the corresponding sheaf. We give another characterization of Post algebras by regular chain bases.

Throughout this paper, by $\mathrm{L}$ we mean a (nontrivial) bounded distributive lattice $(L, V, \lambda, 0,1)$ and $B=B(L)$ the Boolean algebra of complemented elements of $L$. For any at $B$, we denote the complement of $a$ by $a^{\prime}$. For any $x, y \in L$, we denote the largest $z \in L$ such that $x \wedge z \leq y$ (if it exists) by $\mathrm{x} \rightarrow \mathrm{y}$ and the largest element $\mathrm{a} \in \mathrm{B}$ such that $\mathrm{x} \wedge \mathrm{a} \leq \mathrm{y}$ (if it exists) by $x \Rightarrow y$. If, for every $x, y \in L, x \rightarrow y(x \Rightarrow y)$ exists, then we say that $L$ is a Heyting algebra (respectively $B$ algebra). Dually, we define $x+y$ and $x \neq y$ respectively. If in a Heyting algebra $(B-a l g e b r a),(x+y) \vee(y \rightarrow x)$ $=1((x \Rightarrow y) V(y \Rightarrow x)=1)$ for every $x, y \in I$, then we say that $L$ is an L-algebra 
(respectively BL-algebra). For any $x \in L$, if $x \rightarrow 0$ exists, then we say that $x$ has the pseudocomplement and we usually write $x^{*}$ for $x \rightarrow 0$. If $x^{*}$ exists for each $x \in L$, then we say that $L$ is pseuodcomplemented. The dual of $L$ is denoted by $L^{d}$. If both $L$ and $L^{d}$ are Heyting algebras (B-algebras, L-algebras, BL-algebras), then we say that $\mathrm{L}$ is a double Heyting algebra (respectively double B-algebra, double L-algebra, double BL-algebra). L is said to be a P-algebra if $L$ is a BL-algebra. Epstein and Horn proved that $L$ is a P-algebra if and only if $L$ is a double L-algebra ([6], theorem 3.4). For the elementary properties of these types of lattices, we refer to ([2]) and $([6])$.

By a sheaf of bounded distributive lattices we mean a triple $(\mathcal{Y}, \pi, \mathrm{X})$ satisfying the following:

i) $\mathcal{Y}$ and $x$ are topological spaces

i1) $\pi: Y \rightarrow x$ is a local homeomorphism

iii) $\operatorname{Each} \pi^{-1}(p), p \in X$ is a bounded distributive lattice;

iv) the maps $(x, y) \mapsto x$ b and $(x, y) \mapsto \quad x \wedge y$ from $\mathcal{Y} v\}=\{(x, y) \epsilon$ $\varphi_{x} Y / \pi(x)=\pi(y)$ \} into $\mathcal{Y}$ are continuous and

v) the maps $\hat{0}: p \mapsto O(p)$ and $\hat{1}: p \mapsto 1(p)$ from $X \rightarrow Q$ are continuous, where $O(p)$ and $1(p)$ are the smallest and largest elements of $\pi^{-1}(p)$ respectively.

We call $Y$ the sheaf space $X$ the base space and $\pi$ the projection map. We write $Y_{p}$ for $\pi^{-1}(p), p \in X$ and call $Y_{p}$ the stalk at $p$. By a (global) section of the sheaf $(\mathcal{Y}, \pi, x)$ we mean a continuous map $\sigma: x \rightarrow Y_{\text {such that } \pi \circ \sigma=1 d x}$. For any sections $\sigma$ and $\tau$ we write $|(\sigma, \tau)|$ for the closed set $\{p \in x \mid \sigma(p) \neq \tau(p)\}$ and we call $|(\sigma, 0)|$ the support of $\sigma$ and write $|\sigma|$ for $|(\sigma, 0)|$. For the preliminary results on sheaf theory, we refer to the pioneering work of Hofmann ([8]). 
By Spec L, we mean the (Stone) space $Y$ of all prime ideals of $L$ with the hull-kernel topology; i.e., the topology for which $\left\{Y_{x} \mid x \in L\right\}$ is a base, where for any $x \in L, Y_{X}=\{P \in \operatorname{Spec} L / x \notin P\}$. Throughout this paper $X$ denotes Spec B which is a Boolean space, ie., a compact, Hausdorff and totally disconnected space. Since $a H_{a}$ is a Boolean isomorphism of $B$ onto the Boolean algebra of all clopen subsets of $x$, we identify a and $x_{a}$ and write simply a for $x_{a}$. For any $p \in x, \varphi_{p}$ be the quotient lattice $L / \theta_{p}$ where $\theta_{p}$ is the congruence on $L$ given by

$$
(\mathrm{x}, \mathrm{y}) \in \theta_{\mathrm{p}} \Leftrightarrow \mathrm{x} \wedge \mathrm{a}=\mathrm{y} \wedge \mathrm{a} \text { for some } \mathrm{a} \in \mathrm{B}-\mathrm{p} \text {, }
$$

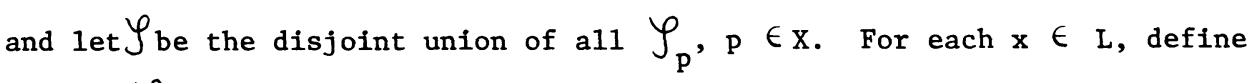
$\hat{x}: x \rightarrow Y_{\text {by }} \hat{x}(p)=\theta_{p}(x)$, the congruence class of $\theta_{p}$ containing $x$. Topologize $Y$ with the largest topology such that each $\hat{x}, x \in L$, is continuous. Define $\pi: Y_{\rightarrow} \mathrm{x}$ by $\pi(s)=p$ if $s \in Y_{\mathrm{p}}$. The following theorem is the main tool used in this paper and is due to Subrahmanyam ([15]) (see also [9]).

THEOREM $1.1(\mathcal{Y}, \pi, \mathrm{X})$ described above is a sheaf of bounded distributive lattices in which each stalk has exactly two complemented elements, viz., $0(\mathrm{p})$ and $1(\mathrm{p})$.

1.2 For any $a \in B, p \in X, \hat{a}(p)=1(p)$ if $p \in a$ and $\hat{a}(p)=0(p)$ if $\mathrm{p} \notin \mathrm{a}$.

1.3 For any $x, y \in L$ and $a \in B, \hat{x} / a=\hat{y} / a$ if and only if $x \wedge a=y \wedge a$.

$1.4 \mathrm{x} \mapsto \hat{\mathrm{x}}$ is an isomorphism of $\mathrm{L}$ onto the lattice $\Gamma(\mathrm{x}, \mathcal{Y})$ of all global sections of the sheaf $(\mathcal{Y}, \pi, \mathrm{x})$. We identify $\hat{x}$ with $\mathrm{x}$ and write simply $x$ for $\hat{x}$.

1.5 For any prime ideal $\mathrm{P}$ of $\mathrm{L}$, there exists a unique $\mathrm{p} \in \mathrm{X}$ such that $\{x(p) / x \in P\}$ is a prime ideal of $\mathcal{Y}_{p}$. On the other hand if $Q$ is a prime ideal of $Y_{p}, p \in X$, then $\{x \in L / x(p) \in Q\}$ is a prime ideal of L. This 
correspondence exhibits the set of all prime ideals of $\mathrm{L}$ as the disjoint union of the sets of prime ideals of the stalks. Moreover, if $P$ and $Q$ are prime ideals of distinct stalks $Y_{p}$ and $Y_{q}$, then $P$ and $Q$ are incomparable, when they are regarded as prime ideals of $L$.

Throughout this paper, by a stalk $\bigcup_{\mathrm{p}}, \mathrm{p} \in \mathrm{x}$, we mean the stalks of the sheaf $(\mathcal{Y}, \pi, \mathrm{x})$ described above at $\mathrm{p}$.

\section{PSEUDOCOMPLEMENTED LATTICES.}

It is well known that a bounded distributive lattice is a Heyting algebra if and only if it is relatively pseudocomplemented; $1 . e$. , each interval $[x, y]$, $\mathrm{x} \leq \mathrm{y} \in \mathrm{L}$ is pseudocomplemented $([1])$. Also the class of all distributive pseudocomplemented lattices and the class of all Heyting algebras are equationally definable ( see [1] and [11]), when we regard the pseudocomplementation and $(x, y) \mapsto(x \rightarrow y)$ as unary and binary operations respectively in L. Thanks to the referee for suggesting a simpler proof of the following.

THEOREM 2.1. L is pseudocomplemtnetd if and only if each stalk $\zeta_{\mathrm{p}}, \mathrm{p}, \mathrm{x}$ is pseudocomplemented and the pseudocomplementation $\mathrm{x} \mapsto \mathrm{x}^{*}$ is continuous and in this case, the pseudocomplement of $x(p)$ in $Y_{p}$ is precisely $x^{*}(p)$ for a11 $\mathrm{x} \in \mathrm{L}$.

PROOF. Suppose L is pseudocomplemented. Then it is easily seen that for all $x$ and $p,(x(p))_{\rho_{p}}^{*}$ exists and is equal to $x^{*}(p)$. Then it is easy to show that the map $x \mapsto x^{*}$ is continuous. For the converse, if $x \in L$, the hypothesis implies that the map $f: x \rightarrow Y$ defined by $f(p)=(x(p))^{*}$ is a global section of $\mathcal{Y}$. Therefore, $f=\hat{y}$ for some $y$ and it is clear that $y=x^{*}$.

If $L$ is a Heyting algebra, then each $\theta_{a}, a \in B$, is compatable with the binary operation $(x, y) \mapsto(x \rightarrow y)$. For, if $a \in B$ and $(x, y)$ and $\left(x_{1}, y_{1}\right) \in \theta$ then $\left(x \rightarrow x_{1}\right) \wedge y \wedge a=\left(x \rightarrow x_{1}\right) \wedge x \wedge a \leq x_{1} \wedge a=y_{1} \wedge a \leq y_{1}$, so that $\left(x \rightarrow x_{1}\right) a \leq$ $\left(y \rightarrow y_{1}\right) \wedge a$. Similarly, we have $\left(y \rightarrow y_{1}\right) \wedge a \leq\left(x \rightarrow x_{1}\right) \wedge a$ and hence 
$\left(x \rightarrow x_{1}, y \rightarrow y_{1}\right) \in \theta_{a}$. Hence the following theorem and its proof are analogous to the above.

THEOREM 2.2. $L$ is a Heyting algebra if and only if each stalk $\zeta_{p}, p \in X$ is a Heyting algebra, and the operation $(s, t) t(s \rightarrow t)$ of $\bigcup_{\mathrm{V}} \jmath_{\text {into }} \zeta_{\text {is }}$ continuous and in this case $x(p) \rightarrow y(p)$ in $\mathcal{J}_{p}, p \in x$, is equal to $(x \rightarrow y)(p)$ for all $x, y \in L$.

\section{NORMAL LATTICES.}

DEFINITION 3.1. (Cornish [4]). L is said to be normal if any two distinct minimal prime ideals of $L$ are comaximal and $L$ is said to be relatively normal if each interval $[x, y], x \leq y \in L$ is normal.

For any $x, y \in L$, let $(x, y)_{L}^{*}$ be the ideal $\{z \in L / x \wedge z \leq y\}$ of $L$. For any $x \in L$, we write $(x)_{L}^{*}$ for $(x, 0)_{L}^{*}$. Cornish $([4])$ proved that $L$ is normal if and only if $(x \wedge y)_{L}^{*}=(x)_{L}^{*} V(y)_{L}^{*}$ for all $x, y \in L$, and that $L$ is relatively normal if and only if $(x \wedge y, z)_{L}^{*}=(x, z)_{L}^{*} V(y, z)_{L}^{*}$ for all $x, y, z \in L$ where $v$ stands for the join operation in the lattice of all ideals of $L$.

THEOREM 3.2. (Speed [13]). A pseudocomplemented distributive lattice is normal if and only if it is a Stone lattice.

THEOREM 3.3. (Balbes and Horn [1]): A Heyting algebra is relatively normal if and only if it is an L-algebra.

THEOREM 3.4. L is normal if and only if each stalk $\varrho_{p}, p \in X$, is normal.

PROOF. Suppose $L$ is normal and $p \in X$. Let $u, v \in Y_{p}$ so that $u=x(p)$

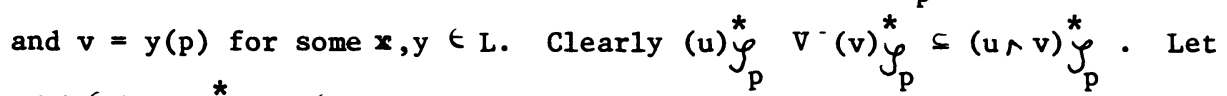
$t(p) \in(u \wedge v)_{\zeta_{p}}^{*}, t \in L$. Since, $(x \wedge y \wedge t)(p)=x(p) \wedge y(p) \wedge t(p)=0(p)$ there exists $a \in B-p$ such that $x \wedge y \wedge t \wedge a=0$, so that $t \in(x \wedge y \wedge a)_{L}^{*}=(x \wedge a)_{L}^{*} v$ $(y \wedge a)_{L}^{*}$ and hence $t=t_{1} \vee t_{2}$ for some $t_{1} \in(x \wedge a)_{L}^{*}$ and $t_{2} \in(y \wedge a)_{L}^{*}$. Now $t(p)=t_{1}(p) v_{t_{2}}(p), t_{1}(p) \in(u)_{\rho_{p}}^{*}$ and $t_{2}(p) \in(v)_{\varphi_{p}}^{*}$. Hence $\bigcup_{p}$ is normal. Conversely, suppose each stalk $\mathcal{J}_{p}, p \in X$ is normal. Let $x, y \in L$ and 
$z \in(x \wedge y)_{L}^{*}$. For each $p \in x$, since $z(p) \in(x(p) \wedge y(p))_{\varphi_{p}}^{*}=(x(p))_{\varphi_{p}}^{*} v(y(p))_{\varphi_{p}^{*}}^{*}$ there exists $a \in B-p, t$ and $s \in L$, such that $a \wedge z=a \wedge(t v s), t \wedge x \wedge a=$ $s \wedge y \wedge a=0$. By the compactness of $x$, it follows that there exists $a_{1}, a_{2}, \ldots$, $\cdot a_{n_{1}} \in B$ and $t_{1}, t_{2}, \ldots, t_{n}, s_{1}, \ldots, s_{n} \in L$ such that $\underset{i=1}{v} a_{1}=1, a_{1} \wedge z=a_{1} \wedge$

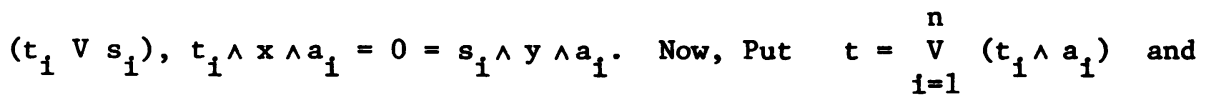

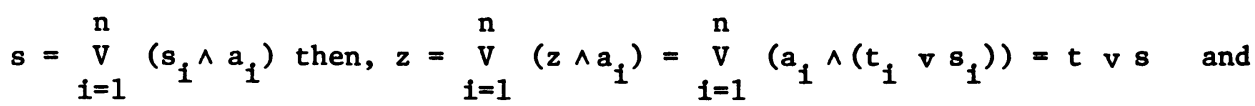
$t \wedge x=v_{i=1}^{n}\left(t_{i} \wedge a_{i} \wedge x\right)=0=v_{i=1}^{n}\left(s_{i} \wedge a_{i} \wedge y\right)=s \wedge y$. Hence $(x \wedge y)_{L}^{*} \varepsilon(x)_{L}^{*} v(y)_{L}^{*}$ and the otherside inclusion is obvious. Hence $L$ is normal.

The proof of the following theorem is analogus to that of the above.

THEOREM 3.5. L is relatively normal if and only if each stalk $\zeta_{p}, p \in x$, is relatively normal.

DEFINITION 3.6. (Speed [12]). L is said to be a distributive * lattice and denoted by $L \in \Delta^{*}$ if, for each $x \in L$, there exists $y \in L$ such that $(\mathrm{x})_{\mathrm{L}}^{* *}:=\left\{\mathrm{u} \in_{\mathrm{L}} / \mathrm{u} \wedge \mathrm{v}=0\right.$ for every $\left.\mathrm{v} \in(\mathrm{x})_{\mathrm{L}}^{*}\right\}=(\mathrm{y})_{\mathrm{L}}^{*}$.

Speed $\left([12]\right.$ proved that $L \in \Delta^{*}$ if and only if, for each $x \in L$, there exists $y \in L$, such that $x \wedge y=0$ and $x \vee y$ is dense; i.e., $(x \wedge y)_{L}^{*}=\{0\}$. THEOREM 3.7. $L \in \Delta^{*}$ if and only if (i) $Y_{p} \in \Delta^{*}$ for each $p \in X$ and (ii) $\left\{p \in X \quad x(p)\right.$ is dense in $\left.Y_{p}\right\}$ is open for each $x \in L$.

PROOF. Suppose $L \in \Delta^{*}$ and $x \in L$. There exists $y \in L$ such that $x \wedge y=0$ and $x \vee y$ is dense in L. Let $p \in x$. Clearly, $x(p) \wedge y(p)=0(p)$. Also, if $z \in L$, such that $((x(p) \vee y(p)) \wedge z(p)=0(p)$, then $(x \vee y) \wedge z \wedge a=0$ for some $a \in B-p$ and hence $z \wedge a=0$, so that $z(p)=0(p)$. Hence $x(p) \vee y(p)$ is dense in $Y_{p^{*}}$. Therefore $Y_{p} \in \Delta^{*}$. Now, suppose $x(p)$ is dense in $Y_{p}$. It follows that $y(p)=0(p)$ and hence there exists $a \in B-p$ such that $y \wedge a=0$. 
We claim that $x(q)$ is dense in $Y_{q}$ for all $q \in$ a. For, if $q \in$ a and $z(q) \in Y_{q}$, $z \in L$, such that $x(q) \wedge z(q)=0(q)$, then there exists $b \in B-q$ such that $x \wedge z \wedge b=0$; so that $(x \vee y) \wedge z \wedge a \wedge b=0$, and hence $z \wedge a \wedge b=0$ and since $a \wedge b \in B-q, z(q)=0(q)$. Conversely, suppose (i) and (ii) hold and $x \in L$. For each $p \in X$, by ( $i$ ) and (ii), there exists $y \in L$ and $a \in B-p$ such that $x \wedge y \wedge a=0$ and $(x \vee y)(q)$ is dense in $Y_{q}$ for all $q \in a$. By the usual compactness argument, there exists $y_{1}, y_{2}, \ldots, y_{n} \in L, a_{1}, \ldots, a_{n} \in B$ such that $\mathrm{v}_{i=1}^{n} a_{i}=1, a_{i} \wedge a_{j}=0$ for $i \neq j, x \wedge y_{i} \wedge a_{i}=0$ and $\left(x \vee y_{i}\right)(p)$ is dense in $Y_{p}$ for all $p \in a_{i}$. Now put $y=v_{i=1}^{n}\left(y_{i} \wedge a_{i}\right)$. Then $x \wedge y=0$ and $x \vee y$ is dense in L. For, $(x \vee y) \wedge z=0$ for some $z \in L$, then, for all $p \in a_{i}$, $0(p)=((x \vee y) \wedge z)(p)=(x(p) \vee y(p)) \wedge z(p)$ and hence $z(p)=0(p)$ for all $p \in a_{i}$ and therefore $z=0$. Hence $L \in \Delta^{*}$.

\section{STONE LATTICES.}

For any $\mathrm{p} \in \mathrm{X}$, since the stalk $\bigcup_{\mathrm{p}}$ has exactly two complemented elements, $Y_{p}$ is a stone lattice if and only if $Y_{p}$ is dense (i.e., if $x(p) \neq o(p)$, then $\left.(x(p)) \stackrel{\star}{\varphi}_{P}=\{0(p)\}\right)$. Hence, by theorem $2.1,3.2$, and 3.4 , we have the following.

THEOREM 4.1. Suppose $\mathrm{L}$ is pseudocomplemented. Then the following are equivalent.

(i) L is a Stone lattice

(ii) L is normal

(iii) Each stalk $Y_{p}, p \in x$, is a normal

(iv) Each stalk $\varphi_{\mathrm{p}}, \mathrm{p} \in \mathrm{X}$, is a Stone lattice

(v) Each stalk $Y_{\mathrm{p}}, \mathrm{p} \in \mathrm{X}$, is dense.

The following theorem is a consequence of theorem $2.2,3.3$ and 3.5 . 
THEOREM 4.2. Let $\mathrm{L}$ be a Heyting algebra. Then the following are equivalent.
(i) L is an L-algebra
(ii) L is relatively normal
(iii) Each stalk $Y_{p}, p \in x$, is relatively normal
(iv) Each stalk $Y_{\mathrm{p}}, \mathrm{p} \in \mathrm{X}$, is an L-algebra.

Since $\mathrm{L}$ is an L-algebra if and only if it is relatively Stone lattice (Theorem 4.11 of [1]) (i.e., each interval is a Stone lattice) in view of theorem 4.1, one may suspect that if $\mathrm{L}$ is an L-algebra, then each stalk is relatively dense and hence a chain. This is not true (see 4.4 below), though the converse is proved in the following.

THEOREM 4.3. If $\mathrm{L}$ is a Heyting algebra and each stalk is a chain, then $\mathrm{L}$ is an L-algebra.

PROOF. If each stalk is a chain, then by theorem 1.5, the prime ideals of $\mathrm{L}$ lie in disjoint maximal chains and hence $\mathrm{L}$ is relatively normal lattice and hence the theorem follows from theorem 2.3.

EXAMPLE 4.4. Let $\mathrm{B}_{4}$ be the 4-element Boolean algebra and $\mathrm{A}$ be the distributive lattice obtained by adjoining an external element to $B_{4}$ as the smallest element. Then A is an L-algebra which is not a chain (Thanks to the referee).

Epstein and Horn $([6])$ proved that $L$ is a Stone lattice if and only if $L^{d}$ is pseudosupplemented and $0 \Leftarrow \mathrm{x} \wedge \mathrm{y}=(0 \Leftarrow \mathrm{x}) \wedge(0 \Leftarrow \mathrm{y})$ for all $\mathrm{x}, \mathrm{y} \in \mathrm{L}$. Now, these two necessary and sufficient conditions for $L$ to be a Stone lattice can be viewed in terms of the stalks as follows.

THEOREM 4.5. $L^{d}$ is pseudosupplemented if and only if $|x|$ is open for each $\mathrm{x} \in \mathrm{L}$ and in this case $|\mathrm{x}|=0 \Leftarrow \mathrm{x}$ for all $\mathrm{x} \in \mathrm{L}$.

PROOF. Follows from Lemma 5.2. 
For any $p \in X$, let $(p)$ be the smallest ideal of $L$ containing $p$. The proof of the following theorem is simple.

THEOREM 4.6. For any $p \in x$, the stalk $Y_{p}$ is dense if and only if ( $p$ ) is a prime ideal of $\mathrm{L}$.

It can be easily seen that each stalk $\mathcal{Y}_{p}, p \in X$, is dense if and only if $|x \wedge y|=|x| \cap|y|$ for all $x, y \in L$. Hence from theorem 4.5 and 4.6 and lemma 2.9 of ( $[7]$ ), we have the following.

THEOREM 4.7. L is a Stone lattice if and only if $|x|$ is open for all $x \in L$ and each stalk $Y_{p}, p \in X$ is dense.

REMARK 4.8. Swamy and Rama Rao ( $[10]$ proved that a lattice $\mathrm{L}$ is a Stone lattice if and only if $\mathrm{L}$ is isomorphic to the lattice of all global sections of a sheaf of dense bounded distributive lattices over a Boolean space in which each section has open support (see also [9] ). It can be verified, that when $L$ is a Stone lattice, then our sheaf $(\zeta, \pi, X)$ coincides with the sheaf constructed in $([10])$.

\section{P-ALGEBRAS.}

The following results interpret B-algebras in sheaf theoretic terminology.

LEMMA 5.1. Let $x, y \in$ L. Then $x \Rightarrow y$ exists in $B$ if and only if $\{p \in X / x(p) \leq y(p)\}$ is closed and in this case $x \Rightarrow y=\{p \in x / x(p) \leq y(p)\}$.

PROOF. For any $p \in X, x(p) \leq y(p)$ if and only if there exists $a \in B-p$ such that $x \wedge a \leq y$. If $x \Rightarrow y$ exists in $B$, then, for any $p \in x, x(p) \leq y(p)$ if and only if $p \in x \Rightarrow y$. Conversely, if $\{p \in x / x(p) \leq y(p)\}$ is closed, then there exists $a \in B$ such that $p \in a$ if and only if $x(p) \leq y(p)$ for all $p \in x$. Hence $a=x \Rightarrow y$.

The proof of the following is easy.

LEMMA 5.2. For any $x, y \in L,|(x, y)|$ is open if and only if there exists a largest element $a$ of $B$ such that $x \wedge a=y \wedge a$. 
The following theorem is a consequence of the above lemmas.

THEOREM 5.3. The following are equivalent.

1) L is a dual B-algebra

2) For any $x, y \in L,\{a \in B / x \vee a=y \vee a\}$ is a principal filter of $B$.

3) For any $x, y \in L,\{a \in B / x \wedge a=y \wedge a\}$ is a principal ideal of $B$.

4) $L$ is a B-algebra

5) $\{p \in x / x(p) \leq y(p)\}$ is closed for every $x, y \in L$.

6) $|(x, y)|$ is open for every $x, y \in L$.

THEOREM 5.4. Suppose L is a B-algebra. Then the following are equivalent.

1) $\mathrm{L}$ is a P-algebra; i.e. L is a BL-algebra

2) Each stalk is a chain

3) For every $x, y, \in L$, there exists $a \in B$ such that $x \wedge a \leq y$ and $y \wedge a^{\prime} \leq x$.

4) For every $x, y \in L$, there exists $a \in B$ such that $x \vee a \geq y$ and $y \vee a^{\prime} \geq x$. PROOF. $2 \Leftrightarrow 3$ is proved in ([15]) and $3 \Leftrightarrow 4$ is clear. $1 \Leftrightarrow 2$ follows from lemma 5.1 .

6. POST ALGEBRAS.

The following definition is slightly different from that of Chang and Horn ( [3] ).

DEFINITION 6.1. By a generalized Post algebra, we mean the lattice $C(Z, C)$ of all continuous maps of a Boolean space $\mathrm{Z}$ into a discrete bounded chain $\mathrm{C}$ where, the operations are pointwise.

THEOREM 6.2. The following are equivalent

1) L is a generalized Post algebra.

2) There exists a chain $c$ in $L$ such that the natural map $c r c(p): c \rightarrow J_{p}$ is an isomorphism for all $\mathrm{p} \in \mathrm{X}$.

3) There exists a chain $C$ and, for each $p \in x$, an order isomorphism $\alpha_{p}: c \rightarrow Y_{p}$ such that for any $c \in C$ and $x \in L,\left\{p \in X / \alpha_{p}(c)=x(p)\right\}$ is open in $X$. 
PROOF. $1 \Rightarrow 2$ :

Let $L=C(Z, D)$ where $Z$ is a Boolean space and $D$ is a discrete bounded chain. It is well known that a $\vDash X_{a}$ is a Boolean 1somorphism of the algebra of all clopen subsets of $Z$ onto $B$, the centre of $L$, where $x_{a}$ is the characteristic function on a. We identify $x_{a}$ with $a$. Also the Stone space $X$ is homeomorphic with $\mathrm{Z}$.

Let $C$ be the set of all constant maps of $Z$ into $D$. For any $d \in D$, let $\bar{d}$ denote the constant map which maps every element of $\mathrm{Z}$ onto $\mathrm{d}$. Then $\mathrm{C}$ is a chain in L. Let $p \in X$. Clearly, the natural map $\emptyset_{p}: c \rightarrow \zeta_{p}=L / \theta_{p}$ is a homomorphism.

If $d_{1}, d_{2} \in D$ such that $\bar{d}_{1}(p)=\bar{d}_{2}(p)$ then $\bar{d}_{1} \wedge a=\bar{d}_{2} \wedge$ a for some a $\in B-p$ and hence $d_{1}=d_{2}$. Now, let $x \in L$. Then if $p \in x^{-1}(d)$ for some $d \in D$, since $x: Z \rightarrow D$ is continuous, $x^{-1}(d) \in B-p$ and since $x \wedge x^{-1}(d)=\bar{d} \wedge x^{-1}(d)$, it follows that $(x, \bar{d}) \in \theta_{p}$. Hence $\theta_{p}$ is an isomorphism.

$2 \Rightarrow 3$ : If $C$ is a chain in $L$ and the natural map $\emptyset_{\mathrm{p}}: c \rightarrow \zeta_{\mathrm{p}}$ is an isomorphism for every $p \in X$, then, for any $c \in C$ and $x \in L . \quad\left\{p \in X / \alpha_{p}(c)=\right.$ $x(p)\}=\{p \in X / c(p)=x(p)\}$ which is open.

$3 \Rightarrow 1$ : We'first observe that since $Y_{p}$ is bounded and $\alpha_{p}$ is an isomorphism of $C$ onto $Y_{p}, C$ is also bounded. Let $x=\operatorname{spec} B$. Define $\theta: L \rightarrow C(x, C)$ by $(\theta(x))(p)=\alpha_{p}^{-1}(x(p))$ for each $x \in L$ and $p \in x$. Let $c \in C$. Then

$$
\begin{aligned}
(\theta(x))^{-1}\{c\} & =\left\{p \in X / \alpha_{p}^{-1}(x(p))=c\right\} \\
& =\left\{p \in X / \alpha_{p}(c)=x(p)\right\} \text { is open by (3) and }
\end{aligned}
$$

hence $\theta(x)$ is continuous. Clearly $\theta$ is a homorphism and one-one since $\alpha_{p}^{-1}$ is so. Now, we will show that $\theta$ is onto. Let $f \in C(x, c)$. Define $\sigma: x \rightarrow Y$ by $\sigma(p)=\alpha_{p}(f(p))$ for every $p \in X$. We will show that $\sigma$ is a section. Let $x \in L$ and $a \in B$, then 


$$
\begin{aligned}
\sigma^{-1}(x(a)) & =\left\{p \in a / \alpha_{p}(f(p))=x(p)\right\} \\
& =a \bigcap \bigcup_{c \in C}\{p \in X / f(p)=c\} \Gamma\left\{p \in X / \alpha_{p}(c)=x(p)\right\} .
\end{aligned}
$$

Since $f$ is continusous, it follows that $\sigma^{-1}(x(a))$ is open. Since $\{x(a) / a \in B$ and $x \in L$ \} is a base for the topology on $Y$, it follows that $\sigma$ is continuous and clearly $\pi \circ \sigma=i d x$. Therefore, $\sigma=\hat{x}$ for some $x \in L$ and also $\theta(x)=f$. Hence $\theta$ is an isomorphism and therefore $\mathrm{L}$ is a generalized Post algebra.

THEOREM 6.3. Let $\mathrm{n} \geq 2$ and $\mathrm{L}$ a P-algebra. Then the following are equivalent.

1) L is a Post algebra of order $n$.

2) Spec $\mathrm{L}$ is the disjoint union of maximal chains each with $\mathrm{n}-1$ elements.

3) Each stalk is a chain with $\mathrm{n}$ elements.

PROOF. $1 \Rightarrow 2$ is proved in ( [5]).

Since $L$ is a $P$-algebra, by theorem 4.4, each stalk $Y_{p}, p \in X$, a chain. Also, by theorem $0 .(5)$, Spec $L$ is the disjoint union of the chains spec $Y_{p}, p \in x$.

If Spec $\mathrm{L}$ is the disjoint union of all maximal chains $\mathrm{C}_{\alpha}, \alpha \in \Delta$ each with $\mathrm{n}-1$ elements, then, for any $\mathrm{p} \in \mathrm{x}$, spec $\mathcal{J}_{\mathrm{p}}=\mathrm{c}_{\alpha}$ for some $\alpha \in \Delta$. Hence spec $\bigcup_{\mathrm{p}}$ has $n-1$ elements and therefore $Y_{p}$ has $n$ elements and hence (2) $\Rightarrow(3)$.

Now, suppose each stalk is a chain with $n$ elements and $C_{n}$ is the $n-$ element chain $1<2<\ldots<n$. For any $p \in x$, let $\bigcup_{p}=\left\{0(p)=x_{1 p}(p)<\right.$ $\left.x_{2 p}(p)<\ldots<x_{n p}(p)=1(p)\right\}$ where $x_{1 p}, x_{2 p}, \ldots, x_{n p} \in L$. Define for any $p \in x$, $\alpha_{p}: \quad c_{n} \rightarrow Y_{p}$ by $\alpha_{p}(i)=x_{i p}(p)$ for each $i \in c_{n} \cdot$ clearly, $\alpha_{p}$ is an order isomorphism. Let $i \in C_{n}, x \in L$ and $p \in X$ such that $\alpha_{p}(i)=x(p)$. ie., $x_{i p}(p)=x(p)$ so that there exists $a \in B-p$ such that $x_{i p}(q)=x(q)$ for all $q \in a$. Since $L$ is a B-algebra and $x_{j p}(p)<x_{k p}(p)$ for all $j<k$, by theorem 5.3, there exists $b \in B-p$ such that $x_{j p}(q)<x_{k p}(q)$ for $a l l j<k$ and $q \in b$ and hence $x_{i p}(q)=x_{i q}(q)$ for all $i \in C_{n}$ and $q \in b$. Then $p \in a \wedge b \in B$ and 
for any $q \in a \wedge b, \alpha_{q}(i)=x_{i q}(q)=x_{i p}(q)=x(q)$. Hence $\left\{p \in x / \alpha_{p}(i)=\right.$ $x(p)\}$ is open for each $i \in C_{n}$ and $x \in L$.

DEFINITION 6.4. By a chain base $\mathrm{C}$ for $\mathrm{L}$ we mean a chain $\mathrm{C}$ with 0 in $\mathrm{L}$ such that $L$ is generated by the centre $B$ and $C$; i.e., every $x \in L$ can be

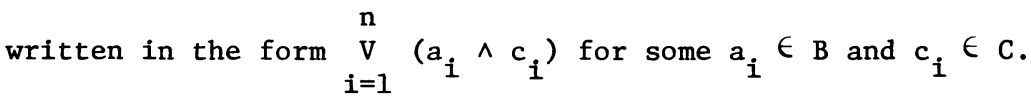

DEFINITION 6.5. A chain base $\mathrm{C}$ in $\mathrm{L}$ is said to be regular, if, for $c_{1} \neq c_{2} \in C$ and $a \in B, c_{1}<c_{2}$ and $a \wedge c_{2} \leq c_{1}$ imply $a=0$.

It is proved in ([15]) that a bounded distributive lattice $L$ is a generalized Post algebra if and only if there exists a regular chain base for L. Now, we characterize chain bases and regular chain bases in terms of the stalks. It is also proved in ([15]) that if $\mathrm{C}$ is a chain base for $\mathrm{L}$, the natural map $\emptyset_{p}: c \rightarrow \zeta_{p}$, defined by $\emptyset_{p}(c)=c(p)$ is an epimorphism for all $\mathrm{p} \in \mathrm{x}$. We prove the converse in the following.

THEOREM 6.6. Let $\mathrm{c}$ be a chain in $\mathrm{L}$ and $0 \in \mathrm{c}$. Then $\emptyset_{\mathrm{p}}: \mathrm{c} \rightarrow \zeta_{\mathrm{p}}$ is an epimorphism for each $\mathrm{p} \in \mathrm{X}$, if and only if $\mathrm{C}$ is a chain base for $\mathrm{L}$.

PROOF. Suppose $\emptyset_{\mathrm{p}}$ is an epimorphism for each $\mathrm{p} \in \mathrm{X}$ and let $\mathrm{x} \in \mathrm{L}$. For each $p \in x$, there exists $c_{p} \in C$ such that $\emptyset_{p}\left(c_{p}\right)=x(p)$ ie., $c_{p}(p)=x(p)$, so that there exists $a \in B-p$ such that $c_{p} \wedge a=x \wedge a$. Therefore, there exists a partition $a_{1}, a_{2}, \ldots, a_{n}$ of $B$ and $c_{1}, c_{2}, \ldots, c \in C$ such that $c_{i} \wedge a_{i}=$

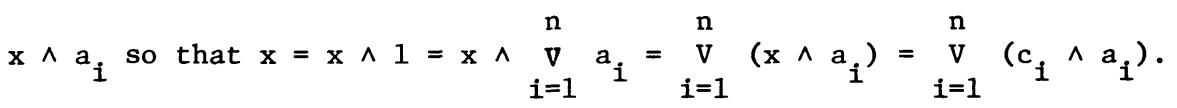
Hence $C$ is a chain base for $L$.

The following theorem is a straight forward verification.

THEOREM 6.7. Let $\mathrm{C}$ be a chain in $\mathrm{L}$. Then the following are equivalent.

1) The natural map $\emptyset_{\mathrm{p}}: \mathrm{c} \rightarrow Y_{\mathrm{p}}$ is one for all $\mathrm{p} \in \mathrm{x}$.

2) For any $c_{1} \neq c_{2} \in C$ and $a \in B, c_{1}<c_{2}$ and $a \wedge c_{2} \leq c_{1}$ imply $a=0$. 
3) For any $c_{1} \neq c_{2} \in C$ and $0 \neq a \in B, a \wedge c_{1} \neq a \wedge c_{2}$.

By summarizing the above results, we have the following :

THEOREM 6.8. Suppose $\mathrm{L}$ is a bounded distributive lattice. Then the following are equivalent.

1) $L$ is a generalized Post algebra

2) There exists a chain $C$ in $L$ such that the natural map $\emptyset_{p}: c \rightarrow Y_{p}$ is an isomorphism for all $p_{l} \in X$.

3) There exists a chain $\mathrm{C}$ and for each $\mathrm{p} \in \mathrm{X}$, an order isomorphism $\alpha_{p}: c \rightarrow Y_{p}$ such that for any $c \in C$ and $x \in L,\left\{p \in X / \alpha_{p}(c)=x(p)\right\}$ is open in $\mathrm{X}$.

4) I has a regular chain base.

REMARK. The equivalence of (1) and (4) is established in ( [15]) by using the Boolean extension techniques.

\section{REFERENCES}

[1] Balbes, R. and Horn, A., "Stone Lattices". Duke Math.J. 37 (1970) 537-545.

[2] Birkhoff, G., "Lattice Theory", Third Edition, AMs. Colloq. Pub1. (1967).

[3] Chang, C.C. and Horn, A., "Prime Ideal Characterization of Generalized Post Algebras", Proc. S ymp. in Pure Maths. Vol. 2, (1961) 43-48.

[4] Cornish, W. H., "Normal Lattices", J. Aust. Math. Soc. 14 (1972), 200-215.

[5] Epstein, G., "Lattice Theory of Post Algebras". Trans. Amer. Math. Soc. 95 (1960) 300-317.

[6] Epstein, G. and Horn, A., "P-algebras an Abstraction of Post Algebras", Algebra Universalis, Vol. 4 (1974) 195-206.

[7] Epstein, G., and Horn, A., "Chain Based Lattices", Pacific J. Maths. Vo1. 55 , No. 1 (1975).

[ 8] Hofmann, K. H., "Representation of Algebras by Continuous Sections". Bull. Amer. Math. Soc. 78 (1972) 291-373.

[9] Maddana Swamy, U., "Representation of Algebras by Sections of Sheaves". Doctoral Thesis, 1974, Andhra University, Waltair, India. 
[10] Maddana Swamy, U., and Rama Rao, V.V., "Triple and Sheaf Representation of Stone Lattices:, Alg. Univ. Vol. $\underline{5}$ (1975) 104-113.

[11] Monterio Antonio, "Axiomes Independents Pour Les Algebras De Brouwer". De Revista de la union Matematica Argentina y da la Asociacion. Fisca Argentiana, Vol. XVII de Homenaje a BEPPO LEVI, 149-160, Buenos Aires, 1955 .

[12] Speed, T. P., "Some Remarks on a Class of Distributive Lattices". J. Aust. Math. Soc. 9 (1969) 289-296.

[13] S peed, T. P., "On Stone Lattices", J. Aust. Math. Soc., 9 (1969) 297-307.

[14] Stone, M. H., "Topological Representations of Distributive Lattices and Brouwerian Logic". Casopis. Pest. Math. Fyz 67 (1937) 1-25.

[15] Subrahmanyam, N. V., "Lectures on Sheaf Theory". Lecture Notes, Dept. of Mathematics, Andhra University, Waltair, India (1978) (unpublished). 


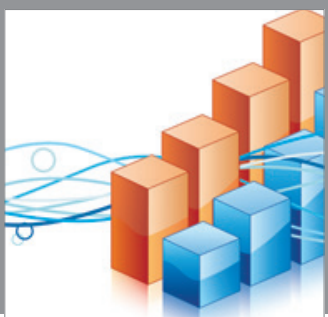

Advances in

Operations Research

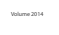

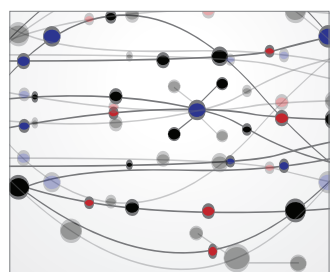

\section{The Scientific} World Journal
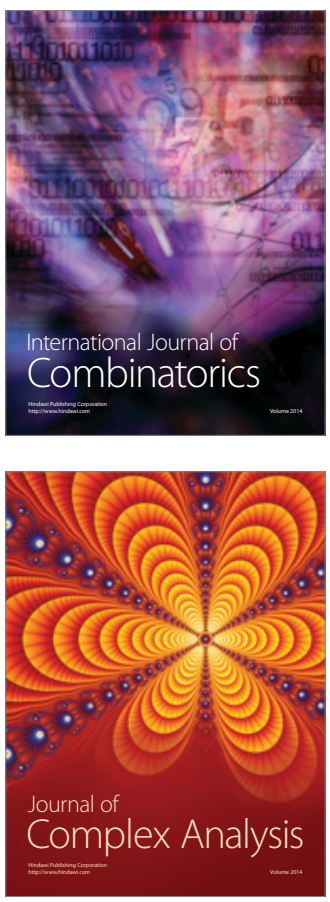

International Journal of

Mathematics and

Mathematical

Sciences
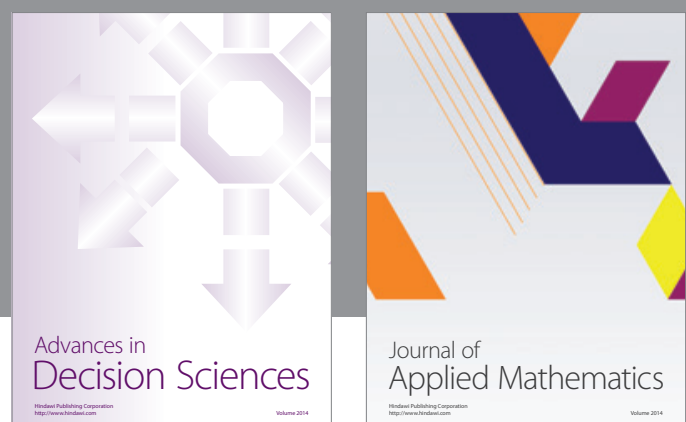

Journal of

Applied Mathematics
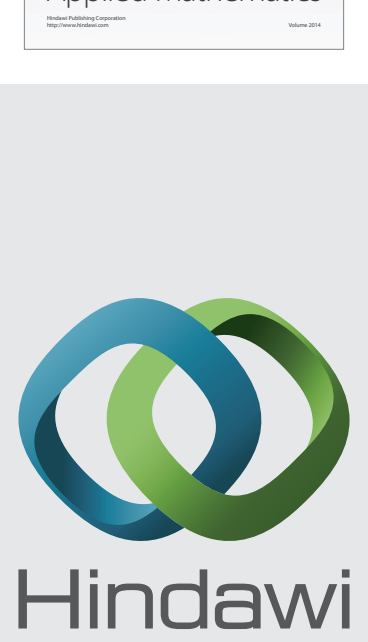

Submit your manuscripts at http://www.hindawi.com
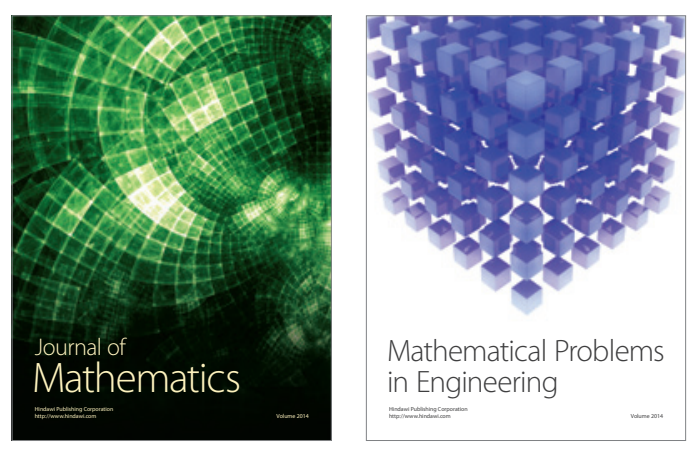

Mathematical Problems in Engineering
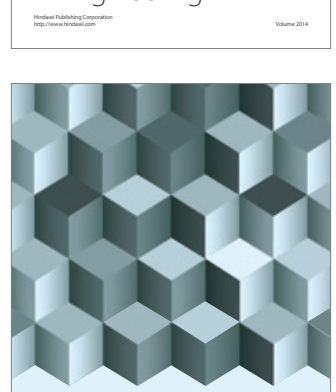

Journal of

Function Spaces
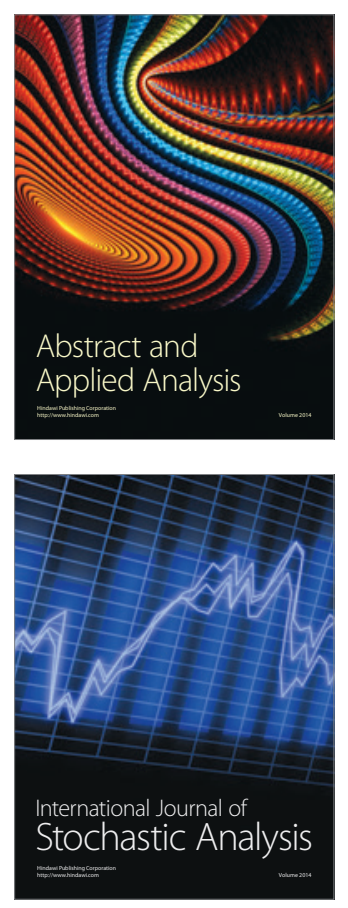

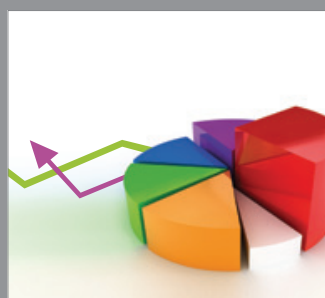

ournal of

Probability and Statistics

Promensencen
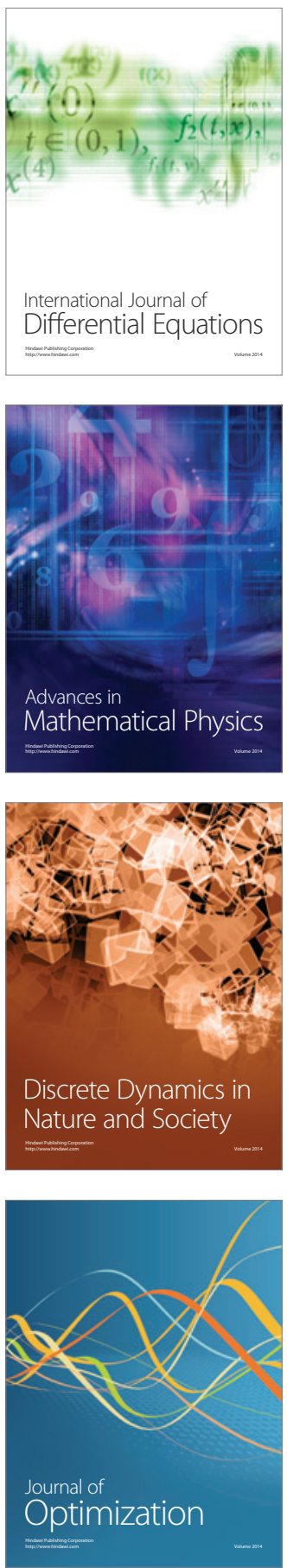